\title{
Developing a Virtual Lab to Teach Essential Biology Laboratory Techniques
}

\author{
Mao Miyamoto, Deborah M. Milkowski, Christine D. Young, and Leah A. Lebowicz
}

Laboratory classes have consistently played a crucial role in science education for many years. Common to all labs is the need to understand essential lab techniques. Students often lack this foundational understanding, and this can lead to poor performance or confidence (Gallagher et al. 2008).

Virtual labs have been found to be effective in promoting active learning and increasing performance (Lewis 2014). In this project, a virtual lab for preparing a phosphate-buffered saline solution (PBS) was created to educate undergraduate biology students on essential laboratory techniques. The virtual lab included animations and interactive elements to visually communicate each step.

Content experts provided input on the accuracy of the scientific content throughout development. Focus group testing with biology teaching assistants (TAs) at the University of Illinois at Chicago was conducted to assess the potential effectiveness of the virtual lab.

\section{OPEN ACCESS}

\section{Keywords}

Virtual lab, essential lab techniques, biology lab, solution preparation, virtual environment, 3D interactivity

\section{Introduction}

Biological research laboratories (labs) associated with undergraduate and graduate biology classes have been playing an important role in higher science education for decades. Hofstein and Lunetta (1982) argue that labs provide a unique environment for teaching and learning science. Their uniqueness lies principally in providing students with opportunities to engage in the processes of investigation and inquiry. (Hofstein and Lunetta, 1982) It is during the lab experience that science becomes alive, helping students to make stronger connections and develop a deeper understanding of the theory and science behind the experiment. (Clough 2002) Studies have shown that learning in the lab occurs when teachers provide students with sufficient time and opportunities for interaction and reflection. (Gunstone and Champagne 1990) Opportunities that prepare students for lab so that they can be more actively engaged during lab time have a vital role in increasing their learning. (Bennett and O’Neale 1998; Carnduff and Reid 2003)

In college level science education, students use instruments such as micropipettes, $\mathrm{pH}$ meters, and electronic balances repeatedly in lab. Students are taught how to operate these instruments as well as how to use them in different lab scenarios; these are commonly referred to as lab skills and mastery of these are crucial to student success. Gallagher and Wiley (2008) state that the "techniques that students typically encounter in college and graduate-level biology courses" are "essential laboratory techniques" that will provide a strong foundation for lab competency, increased performance and safety. (Gallagher and Wiley 2008) Students are commonly required to undergo training on lab safety and essential lab techniques prior to attending any lab session.

Gallagher and Wiley (2008) argue that when essential lab techniques are not clearly taught and students do not possess a strong foundation of these skills, the key principles that underlie important research concepts may often be taken for granted. Teachers should ideally teach lab techniques one on one, but due to the nature of limited working space in the lab and the ratio of instructor to students, this is not always possible. Teaching a technique with a small number of students is ideal because the working space when demonstrating the technique is smaller. In large groups, students often find it difficult to adequately observe all the techniques demonstrated, which can cause a decrease in lab confidence and the requisite skillset. This may cause students to fall behind and make their lab experiences overwhelming. (National Research Council 2006).

Maldarelli (2009) indicates the importance of the problems with teaching students essential lab techniques below:

\footnotetext{
"Students who have never performed a technique must first be guided through the process; thus, a visual demonstration of laboratory procedures is a key element in teaching pedagogy. Current practice in our General
} 


\begin{abstract}
Biology Laboratory Class I and II at Johns Hopkins
University (JHU) has students observe demonstrations of

lab techniques in the teaching lab. Under those conditions,

viewing is inadequate because of space constraints and

there is limited opportunity for review of the technique.

The success of laboratory learning is directly related to

how well the experiment is performed using a standard

laboratory protocol; therefore, preparation for the

laboratory can increase the success rate of the experiment

as well as result in active-learning outcomes. While

students can read about a procedure before class,

typically there is no resource available to view the

technique or procedure beforehand."
\end{abstract}

As technology continues to contribute to advances in education, there has been an emergence of virtual labs that have dramatically changed the education landscape. (Scanlon 2002; Brinson 2015) Research shows that virtual labs are as effective as traditional labs, facilitating an active-learning environment that allows students to learn material at their own pace, thereby, improving students' performance, knowledge, and confidence. (Dobson 2009; Gibbons 2004; Sancho 2006). $3 \mathrm{D}$ visualizations can enhance learning by helping users better understand spatial relationships. (Ma 2010)

Hofstein and Lunetta (2002) state that laboratory experiences have been reported to promote key science education goals including the enhancement of students' understanding of (1) scientific concepts, (2) scientific practical skills, problem solving abilities, and (3) the method of scientific inquiry and reasoning. Virtual labs can facilitate the understanding of (1) scientific concepts and (2) the method of scientific inquiry and reasoning. This is supported by the finding that virtual lab allows students, "to 'stop the world' and 'step outside' of the simulated process to review and understand it better." (Parush 2002) Virtual labs can also be used as tools to better prepare students before entering traditional labs, by increasing their knowledge and confidence. (Crisp 2012; Cunningham 2006; Karamanos 2012) As a virtual lab environment is only a reflection of a real lab environment, subsequent practice in a physical lab is necessary to develop further practical skills.

This project was designed to create a virtual lab for preparing phosphate-buffered saline (PBS) to help undergraduate students understand the appropriate use of essential lab techniques and instruments used in its preparation. The PBS lab was chosen because it contains several basic lab techniques that are commonly used in many biological experiments. In addition, feedback from biology TAs at the University of Illinois at Chicago was obtained on the use of the virtual lab as a possible teaching tool to improve students' knowledge, skill level, performance, and confidence. The virtual PBS lab was used as the context to introduce essential skills and techniques. Animations were included to visually explain techniques and to highlight information to keep in mind when performing these different tasks. In this research, a virtual lab is defined as an interactive program presented on a $2 \mathrm{D}$ platform such as a website and does not include augmented reality (AR), virtual reality (VR) or extended reality (XR).

\section{Essential Laboratory Techniques}

Preparing a solution is one of the most important and basic skillsets in biology and chemistry labs. Solutions are made frequently and a properly made solution is greatly related to the success of the experiment. The protocol of making a solution generally consists of value calculation, value measurement and $\mathrm{pH}$ adjustment. Gallagher and Wiley (2008) speak about the importance of establishing a strong foundation in basic lab skills in the book "Current Protocols Essential Laboratory Techniques." (Gallagher \& Wiley, 2008). They claim that the general descriptions of lab techniques that students typically encounter in college and graduate-level biology courses are typically taken for granted by instructors. They also discuss the importance of proper training and teaching of the theory behind basic techniques as this eases the transition to more sophisticated techniques. This allows beginning researchers to adopt a much more sophisticated approach to experimental design and troubleshooting, two areas that are particularly challenging to beginners who may not fully understand the underlying principles of the techniques that they are using the first three chapters of the book focus on measurement techniques and reagent preparation. The book also expands on the importance of accuracy in weighing, pipetting and $\mathrm{pH}$ adjustment for reagent preparation.

\section{Overview of Solution Preparation Lab for Phosphate-buffered saline}

In this project, animations were created to visualize the preparation of 10X PBS in a virtual lab. PBS is a buffer solution commonly used in biological research because it is isotonic and non-toxic to most cells. The uses for this solution include substance dilution and cell rinsing. Procedures in the PBS lab cover several essential lab skills, including weight calculation, weighing powder, measuring liquid reagents, and $\mathrm{pH}$ adjustment.

Step I Weight Calculation
\begin{tabular}{|l|l|l|l|}
\hline Chemical & $\begin{array}{l}\text { Final } \\
\text { Concentration }\end{array}$ & $\begin{array}{l}\text { Molecular } \\
\text { weight }\end{array}$ & $\begin{array}{l}\text { Grams } \\
\text { Required }\end{array}$ \\
\hline $\mathbf{N a C l}$ & $1.3 \mathrm{M}$ & $58.44 \mathrm{~g} / \mathrm{mol}$ & $75.97 \mathrm{~g}$ \\
\hline $\mathbf{N a H}_{2} \mathbf{P O}_{4}$ & $70 \mathrm{mM}$ & $137.99 \mathrm{~g} / \mathrm{mol}$ & $9.66 \mathrm{~g}$ \\
\hline $\begin{array}{l}\mathbf{N a}_{2} \mathbf{H P O}_{4} \\
\mathbf{1 2 H 2 O}\end{array}$ & $30 \mathrm{mM}$ & $338.14 \mathrm{~g} / \mathrm{mol}$ & $10.74 \mathrm{~g}$ \\
\hline
\end{tabular}

Table 1 Recipe for 1L 10X PBS (pH7.4) 
Students learn the concept of molarity and how to calculate weights required for each reagent. In undergraduate lab sessions, students need to calculate the weight by themselves. This section highlights the importance of moles and molar mass when preparing a solution and understanding the chemistry behind it. (Sambrook 1989)

\section{Step II Weight Measurement and Dissolution}

Students weigh out the appropriate amount of each reagent based on the result of weight calculation. This step involves basic techniques of weighing powdered reagents using an electric balance and dissolving them in deionized water using a magnetic stirrer (Sambrook, 1989).

\section{Step III pH Adjustment}

A buffer solution is a mixture of a weak acid and its conjugate base, or vise versa. Its $\mathrm{pH}$ changes very little when you add a small or moderate amount of strong acid or base to it and is therefore used in experiments to maintain the $\mathrm{pH}$ of a solution.

Being able to properly adjust the $\mathrm{pH}$ of a solution is a key to making a buffer solution. In the PBS lab, students use $\mathrm{HCl}$ and $\mathrm{NaOH}$ solutions to adjust the $\mathrm{pH}$ of the solution (from around 5.3) to 7.4. This step demonstrates proper skills in calibrating a $\mathrm{pH}$ meter, measuring $\mathrm{pH}$, and adding liquid reagents using a micropipette. (Sambrook 1989)

\section{Virtual Environment and 3D Interactivity}

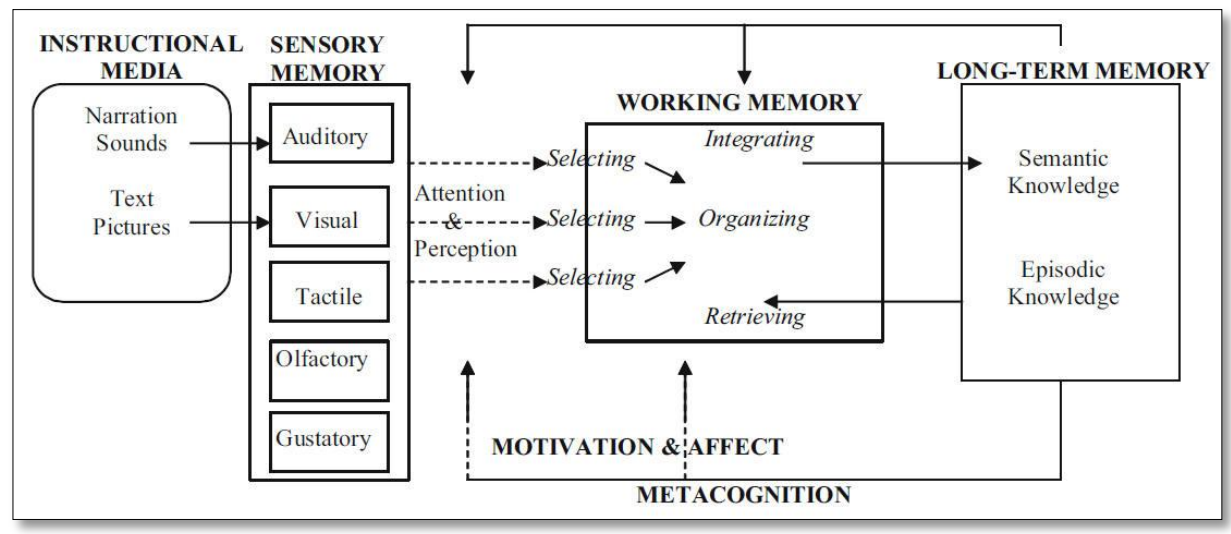

Wann and Williams (1996) define a 3D virtual environment as an environment that "capitalizes upon natural aspects of human perception by extending visual information in three spatial dimensions" and "enables the user to interact with the displayed data" Dalgarno (2010) states the benefits of 3D virtual learning environments (VLEs) as the following; "3-D VLEs can be used to facilitate learning tasks that lead to the development of enhanced spatial knowledge representation of the explored domain." 3-D VLEs can be used to facilitate learning tasks that lead to improved transfer of knowledge and skills to real situations through contextualization of learning (Dalgarno, 2010). Therefore, a virtual learning environment allows students to potentially follow instructions step by step, to work at their own pace, to make mistakes in a safe place, and to repeat the procedures until better understood.

In a cognitive affective theory of learning with media (Figure
Figure 1. A cognitive-affective theory of learning with media. (Moreno and Mayer 2007)

1), Moreno and Mayer (2007) highlight five principles of instructional design in regard to interactive learning: Guided activity, reflection, feedback, control and pre-training. They discuss their principles as follows:

\begin{abstract}
“... interactivity and multimodal presentations do not cause learning, but rather that there are a growing set of research-based principles for using interactive multimodal learning environments in ways that promote learning. A continuing challenge for instructional designers who develop interactive multimodal learning environments is to reduce the extraneous cognitive processing that can be caused by interactivity and to maximize the learner's motivation to engage in generative cognitive processing that can be fostered by interactivity."
\end{abstract}

In order to best incorporate these principles in instructional media, they suggest including the following: games and simulations, pedagogical agents (which can be referred to as guidance), case-based learning, and embedded authentic assessment (in other words, performance assessment).

\section{Virtual Laboratories}

The emergence of virtual labs has dramatically changed the laboratory education landscape. (Scanlon 2002; McAteer 1996) Following the growth of virtual labs, a long-running debate about virtual vs. traditional labs has been unfolded. (Ma 2006) Some research shows that virtual labs are as effective as traditional labs by facilitating active, inquiry-based learning rather than the protocol driven learning normally found in traditional laboratories. (Dobson 2009; Gibbons 2004; Sancho 2006) Lewis (2014) also claims that virtual labs can enable students to learn at their own pace, overcome health and safety constraints, as well as ethical issues. (Lewis 2014) On the other hand, arguments against virtual labs state that data generated in the virtual world lack biological variation and therefore students will not be able to 
analyze, interpret, and learn from trial and error. (Grant 1995; Dobson 2009) They also claim that virtual labs do not provide the hands-on experience of learning individual techniques or the use of real equipment. (Grant 1995; Dobson 2009) The discussion is converging on a conclusion that virtual labs should be integrated into the traditional lab to promote students' learning of science in the twenty-first century (Lewis, 2014). They also suggest using virtual labs in parallel to traditional labs to supplement, reinforce, and enhance the learning between the two (Lewis 2014, Macaulay 2009).

\section{Methodology}

\section{Preproduction}

An animation providing an overview of the developed virtual lab can be viewed at: https://vimeo.com/308142775.

Several lab protocols were used as references to create the final lab protocol for this research. These references included lab protocols from Sambrook (1989) and Fukami-Kageyama laboratory at Kobe University in Japan.

A wireframe was developed to organize the flow of the virtual lab. (Figure 2) Sections are divided to allow for user-guided learning. Students can choose to learn the material in sequence or jump to sections specific to each step. They also have the

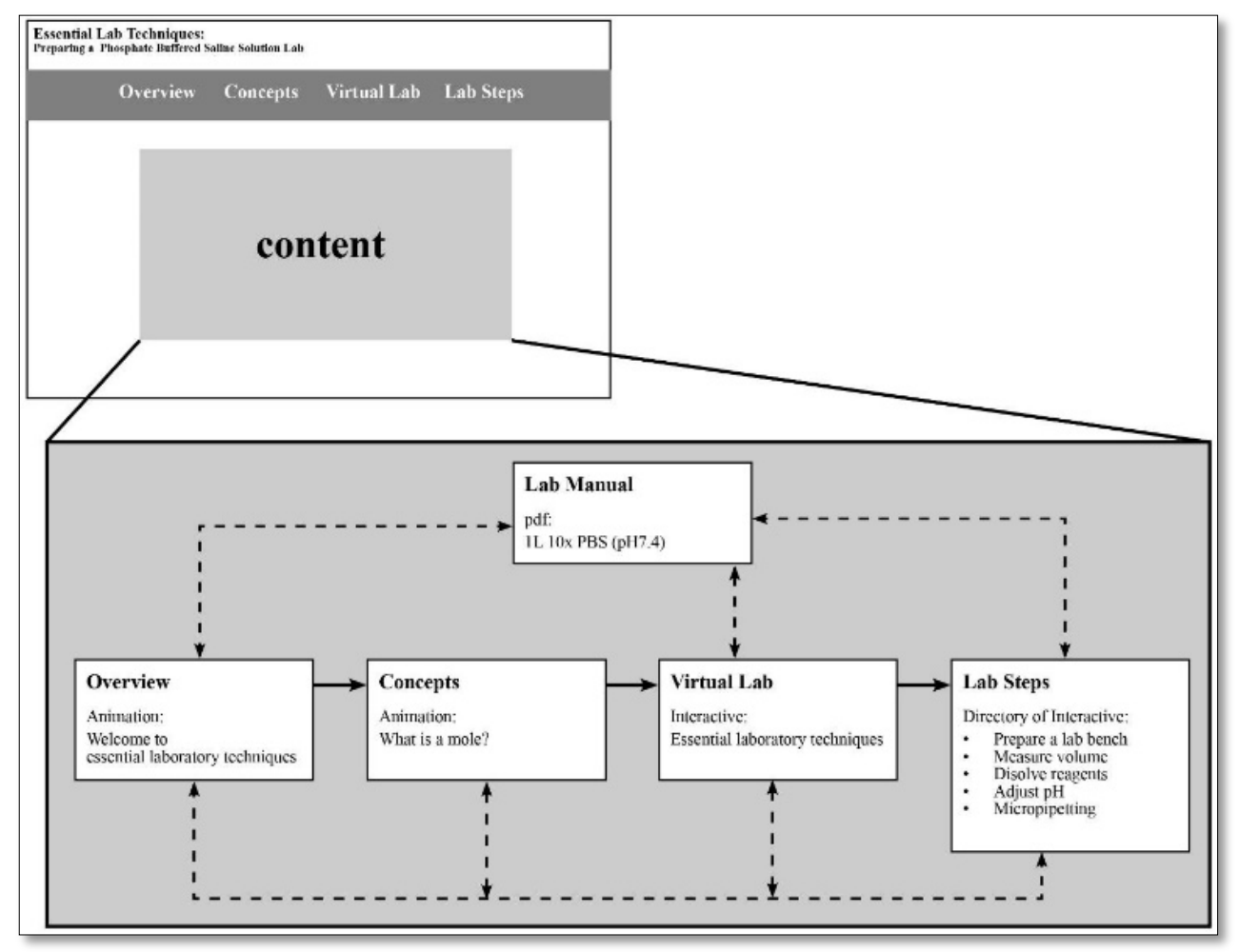

Figure 2 Wireframe of the website option to visit the lab manual at any point to refer to all the information laid out in a document.

\section{Overview}

This section serves as a welcome page and includes the animation "Welcome to Essential Laboratory Techniques." This animation introduces the virtual lab, provides basic information on PBS, and describes what students are expected to learn in the lab.

\section{Concepts}

After visiting the Overview page, the lab directs the users to the Concepts page, which contains animations that teach students scientific concepts they should know before they enter the lab. For this project, the animation "What is a mole?" was created and included in this section. This animation describes what a mole is, how the information in the periodic table is used to determine moles, and how molarity is calculated.

\section{Virtual Lab}

The Virtual Lab page contains the interactive program that walks through the entire experiment. This virtual lab is designed to help students understand the flow of the experiment while learning the details of each step at the same time.

\section{Lab Steps}

Following the Virtual Lab section, Lab Steps contains five interactive programs that were extracted and separated from the virtual lab. The five programs include: "Prepare a lab bench," "Weighing reagent" "Dissolve reagent," "Adjust $\mathrm{pH}$," and "Micropipetting." On this page, students can pick a specific technique to focus on and become familiar with.

Two animations "Welcome to Essential Laboratory Technique" and "What is a mole?" were created for the virtual lab. Storyboards were developed using Adobe Illustrator ${ }^{\circledR}$, Autodesk 3D Studio Max ${ }^{\circledast}$, and Adobe InDesign ${ }^{\circledast}$, to plan out the detailed motion and script in the animations. Scripts and the in progress animation were shown to the content advisor and edited to be scientifically accurate. 


\section{Development of 3D Models}

The lab instruments and lab environment used in the virtual lab and animations were created using Autodesk 3D Studio Max. (Figure 3) This software was chosen because it is very powerful and has a lot of flexibility in terms of creating models, textures, animations, and maps. Photo references were used to create the model.

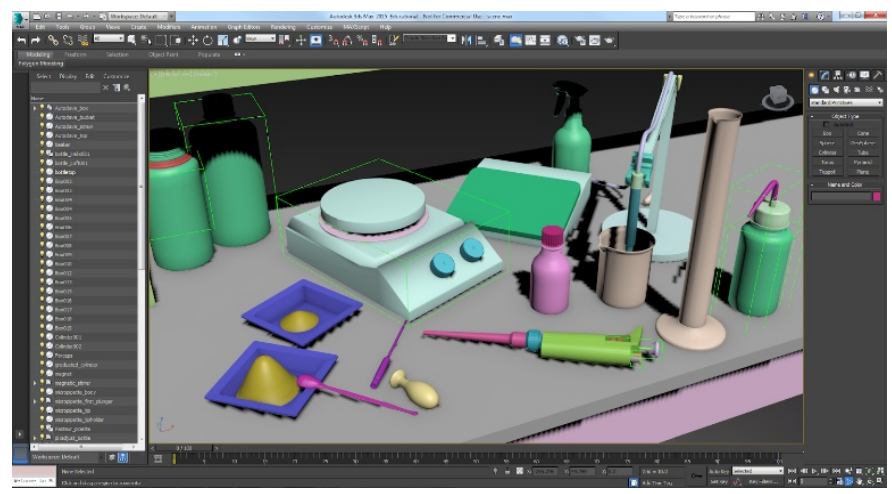

Figure 3. Models of lab environment in 3D Studio Max.

UV maps were created for each model to allow for the application of texture maps. Glass, plastic, and metal textures were created using Arch and Design materials. (Figure 4) (Fig.4). All the models were lit in 3D Studio Max and complete texture maps were made to be imported into Unity.

These maps were also used as a reference to create labels on the bottles, scale on the cylinder, and buttons on the instruments. Labels were made as vector illustrations using Adobe Illustrator. The illustrations were put in appropriate position by layering the illustration on top of the texture map in Adobe Photoshop ${ }^{\circledR}$.

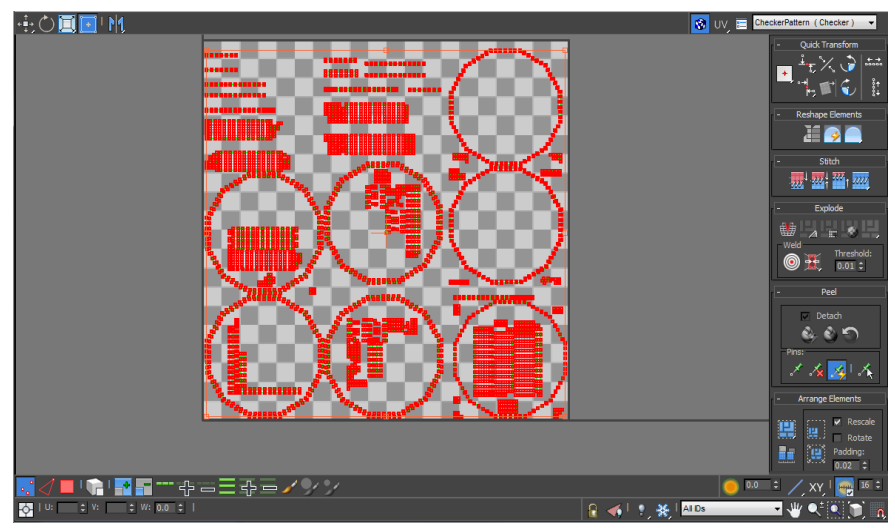

Figure 4 Unwrapped UV of a bottle top

Ink and paint material was applied to all the models for the use in the animations. (Figure 5) Using mental ray renderer, png sequences were rendered for use in the animation.

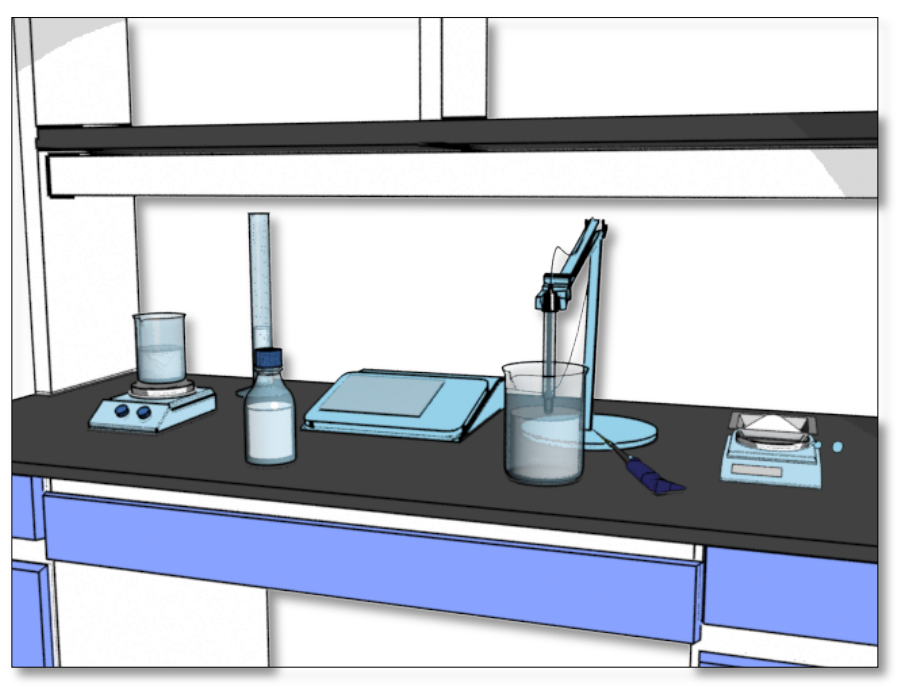

Figure 5 Scene rendered with Ink and Paint material

\section{Development of Animation}

2D animation techniques were used to simplify the content and make it accessible to students.

To achieve this, visual elements were created using Adobe Illustrator and Autodesk 3D Studio Max. Ink and paint materials were applied to the $3 \mathrm{D}$ models so that they resembled 2D illustrations. Many of the visuals were adopted from images used for the storyboard.

Paige Blumer recorded the voiceover using Garage Band ${ }^{\circledR}$. Sound was edited and converted into a proper extension to be further used in the animation using Adobe Audition ${ }^{\circledR}$.

Visuals and voice overs were imported to Adobe After Effects $^{\circledR}$ for compositing. Text and paths were created in After Effects. The, "Welcome to Essential Laboratory Techniques," animation is 30 seconds long and the, "What is a mole?" animation is 1 minute and 30 seconds. The animations were kept short to simplify and clarify the information presented.

\section{Development of Interface Design}

User interfaces were developed to provide easy and logical flow of information to the users. Instructions for each lab step appear in the box in the bottom right corner. A left arrow button always exists to the left of the instructions to allow a user to go back and reset the scene. When a user completes the lab steps, a right arrow appears on the right side of the instruction and directs the user to the next scene. The rest of the screen represents the lab environment to give users a realistic feel of how the lab looks. 


\section{Programming}

All the models, texture maps, and labels were imported to the game engine Unity. The apparatuses were laid on the bench and cameras were created for the scenes. $\mathrm{C \#}$ is used to control the actions. All the codes were made using Sublime Text ${ }^{\star}$. Online forums such as Unity Answers were used to refer and troubleshoot the error with the code. By following the instructions, users can complete the action. Either clicking, dragging, or pressing buttons triggers the actions.

An open-source Unity plug-in "iTween" by PixelPlacement ${ }^{\mathrm{TM}}$ was used to create sequential animation that manipulates position, scale, rotation, and also controls transition.

Display of the electric scale and $\mathrm{pH}$ meter were controlled by creating a random number in a certain range. The number is matched to a texture that is an image of each number. Depending on what number is generated, a texture is assigned to each digit. To pursue reality, the number changes before it settles to a certain number. This is controlled by invoking the function several times before running a function to settle to the final number.

\section{Postproduction}

Html and css were coded using Brackets ${ }^{\circledast}$ and the deployed contents on mmiyam2.people.uic.edu/projectresearch. Buttons and icons were made using Adobe Illustrator and Adobe Photoshop.

Animations were uploaded to $\mathrm{Vimeo}^{\circledR}$ and embedded in the website. The interactive was built for web and also embedded in the website. (Figure 6) In order to run the interactive program, the users require the Unity Webplayer.

\section{Evaluation}

A focus group consisting of 13 teaching assistants who taught biology classes was convened to acquire proof of concept for the virtual lab's potential effectiveness. The participants of the research had various backgrounds, but it should be noted that this focus group was a limited representation of the target population. The evaluation sheet and informed consent forms were distributed before the focus group began. A brief presentation was made to demonstrate the animations and the interactive lab. Process work such as storyboards, user interface design, and experimental design were also shared during the focus group. After the presentation, members discussed the content and asked questions. The focus group discussed how useful the virtual lab could be in promoting students' understanding of basic lab techniques taught, how it may enhance a student's performance on future lab sessions and how to improve the educational design of the lab in the future. Feedback obtained from the focus group was compiled and analyzed.

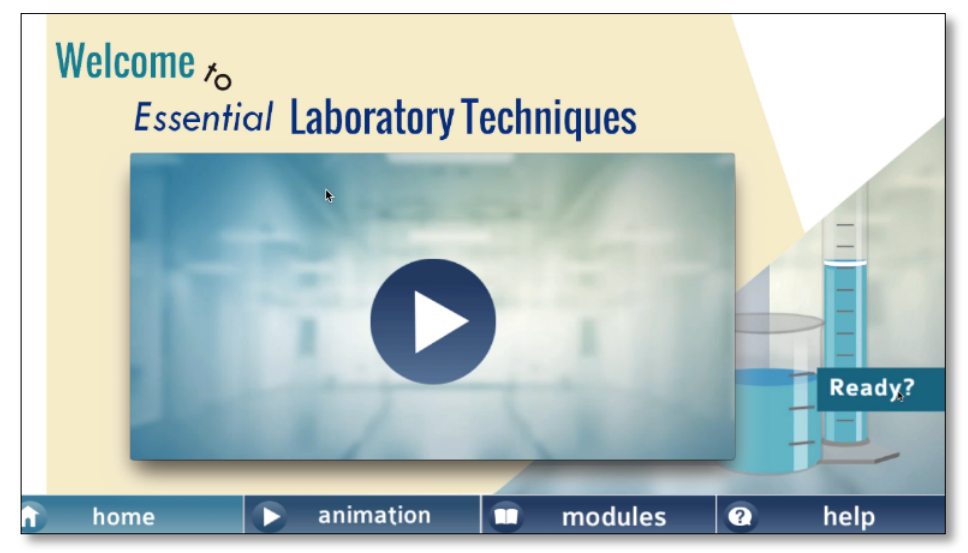

Figure 6. Home screen of the virtual lab

\section{Results}

The ultimate target audience for the application is intended to be undergraduate biology students. In this project, we showed the lab to biology TAs to gain feedback on how we can create a virtual lab to educate undergraduate biology students on essential laboratory techniques.

Thirteen teaching assistants attended the focus group and all of them participated in the discussion and submitted the evaluation sheet. All of the TAs strongly agreed that they would recommend the use of this virtual lab to their students. Also, all of the TAs gave high scores on the question on whether the virtual lab would help increase student performance, knowledge, and confidence in the lab. The TAs reported that they either strongly agreed or agreed that both the virtual lab and the animation have the ability to help students better understand the material. They also indicated that the virtual lab is realistic and visual communication is conveyed successfully through the virtual lab. They found the step-by-step explanation and hands on learning to be helpful for students. They evaluated the introductory animations as good material to show before the virtual lab, and liked the metaphors and framework of the animation.

The TAs mentioned that the order of some of the lab steps and techniques used were inaccurate in the virtual lab. This was because the order was inaccurate in the lab manual which was used as a guide. For the question asking whether the lab was easy to navigate, their scores ranged from strongly agree to disagree. They pointed out that we did not plan the legibility 
well enough, that it was difficult to locate the instructions and that the buttons were hard to read. They also pointed out that the labels on the bottle were hard to read and therefore it was hard to navigate the virtual labs in some scenes. One suggested using a glow to indicate which object students should click on in each step so there is less reliance on the instructions.

Overall, the focus group found the application to be a great resource for students to train and practice both manipulating instruments and the specific laboratory techniques before they attend the lab session. They suggested adding more topics in the future so that the virtual lab may be more comprehensive. For example, some of them suggested adding a section on lab safety. They also suggested including quizzes so students can feel more comfortable about the material. Another participants indicated that there was not enough problem-based learning in the lab and suggested including a math problem to solve for weight calculation.

\section{Discussion}

This virtual lab has the potential to improve student performance and understanding in conducting essential laboratory techniques. From the focus group discussion, we can say that this virtual lab has a strong framework in instructional design. Evaluation suggested that the use of the 3D technique delivered a realistic yet simplified representation of the lab environment, which made the virtual experience a more valuable opportunity for students to familiarize themselves with lab techniques in an easy manner. The five principles of instructional design in interactive learning (guided activity, reflection, feedback, control, and pre-training) were implemented in the virtual lab through step-by-step instruction, feedback unique to the user's action, interactivity, and freedom to explore and make mistakes. The results suggest that the implementation of the five principles of interactive learning was successful in providing a safe place to prepare students. After we make the suggested improvements, we can test the virtual PBS lab on students to evaluate the effectiveness of the visual learning and instructional design. We based the order and techniques of some of the steps in the virtual lab on a lab manual which contained some inaccuracies. We received invaluable feedback and plan to implement it in the next version. The feedback is important because results indicate that the virtual lab could be an effective training tool in many different types of labs that involve solution preparation. It may also have potential to be added as a learning module in a course for students involved in laboratory work in the future.

\section{Acknowledgements}

The authors thank Professor David Featherstone, the Director of Undergraduate Studies of Biological Department at UIC, for his expertise in creating the content, and John Daugherty for his leadership of the UIC Biomedical Visualization graduate program.

\section{References}

Bennett, S.W., O'Neale, K. 1999. Progressive development of practical skills in chemistry: A guide to early-undergraduate experimental work. Royal Society of Chemistry.

Brinson, J.R. 2015. Learning outcome achievement in non-traditional (virtual and remote) versus traditional (hands-on) laboratories: A review of the empirical research. Computers \& Education 87:218-237.

Carnduff, J., and Reid, N. 2003. Enhancing undergraduate chemistry laboratories: pre-laboratory and post-laboratory exercises. Royal Society of Chemistry.

Clough, M.P. 2002. Using the laboratory to enhance student learning. In Learning Science and the Science of Learning, National Science Teachers Association, Editors: R. W. Bybee.

Crisp, K.M. 2012. A structured-inquiry approach to teaching neurophysiology using computer simulation. Journal of Undergraduate Neuroscience Education 11(1):A132.

Cunningham, S.C., McNear, B., Pearlman, R.S., Kern, S.E. 2006. Beverage-agarose gel electrophoresis: An inquiry-based laboratory exercise with virtual adaptation. CBE Life Sciences Education 5(3):281-286.

Dalgarno, B., and Lee, M.J. 2010. What are the learning affordances of 3-D virtual environments? British Journal of Educational Technology 41(1):10-32.

Dobson, J.L. 2009. Evaluation of the virtual physiology of exercise laboratory program. Advances in Physiology Education 33(4):335-342.

Gallagher, S.R., and Wiley, E.A. 2008. Current protocols essential laboratory techniques. Hoboken: Wiley.

Gibbons, N.J., Evans, C., Payne, A., Shah, K., Griffin, D.K. 2004. Computer simulations improve university instructional laboratories. Cell Biology Education 3(4):263-269. 
Grant, A. 1995. The effective use of laboratories in undergraduate courses. International Journal of Mechanical Engineering Education 23(2):95-101.

Gunstone, R.F. and Champagne, A.B. 1990. Promoting conceptual change in the laboratory. In E. Hegarty-Hazel (Ed.). The Student Laboratory and the Science Curriculum. London: Routledge.

Hofstein, A., and Lunetta, V.N. 1982. The role of the laboratory in science teaching: Neglected aspects of research. Review of Educational Research 52(2):201-217.

Hofstein, A., and Lunetta, V N. 2004. The laboratory in science education: Foundations for the twentyfirst century. Science education 88(1):28-54.

Karamanos, K., Gkiolmas, A., Chalkidis, A., Skordoulis, C., Papaconstantinou, M., Stavrou, D. 2012. Ecosystem food-webs as dynamic systems: Educating undergraduate teachers in conceptualizing aspects of food-webs' systemic nature and comportment. Advances in Systems Science and Application 12(4):49-68.

Lewis, D.I. 2014. The pedagogical benefits and pitfalls of virtual tools for teaching and learning laboratory practices in the biological sciences. The Higher Education Academy.

Ma, M., Zheng, H., Lallie, H. 2010. Virtual reality and 3D animation in forensic visualization. Journal of Forensic Sciences 55(5):1227-1231.

Macaulay, J., Van Damme, M., Walker, K. 2009. The use of contextual learning to teach biochemistry to dietetic students. Biochemistry and Molecular Biology Education 37(3):137-142.

Maldarelli, G.A., Hartmann, E.M., Cummings, P.J., Horner, R.D., Obom, K.M., Shingles, R., Pearlman, R.S. 2009. Virtual lab demonstrations improve students' mastery of basic biology laboratory techniques. Journal of Microbiology \& Biology Education: JMBE 10(1):51.

Mayer, R.E., Hegarty, M., Mayer, S., Campbell, J. 2005. When static media promote active learning: Annotated illustrations versus narrated animations in multimedia instruction. Journal of Experimental Psychology: Applied 11(4):256.

McAteer, E., Neil, D., Barr, N., Brown, M., Draper, S., Henderson, F. 1996. Simulation software in a life sciences practical laboratory. Computers \& Education 26(1):101-112.
National Research Council. 2006. America's Lab Report: Investigations in High School Science. Washington, DC: The National Academies Press.

Parush, A., Hamm, H., Shtub, A. 2002. Learning histories in simulation-based teaching: The effects on self-learning and transfer. Computers \& Education 39(4):319-332.

Sambrook, J., Fritsch, E.F., Maniatis, T. 1989 Molecular Cloning: A Laboratory Manual, 2nd ed., volume 3, appendix B.12 Cold Spring Harbor: Cold Spring Harbor Laboratory Press.

Sancho, P., Corral, R., Rivas, T., González, M J., Chordi, A., Tejedor, C. 2006. A blended learning experience for teaching microbiology. Am J Pharm Educ 70(5):120.

Scanlon, E., Morris, E., Di Paolo, T., Cooper, M. 2002.

Contemporary approaches to learning science:

Technologically-mediated practical work.

Wann, J., \& Mon-Williams, M. (1996). What does virtual reality NEED?: Human factors issues in the design of three-dimensional computer environments. International Journal of Human-Computer Studies, 44(6), 829-847.

\section{Authors}

Mao Miyamoto, M.S.(Bio), M.S.(BVIS), CMI is a medical illustrator and science communicator. Originally from Japan, she received a Master of Science in Biology at Kobe University. Mao came to the United States to expand her interest in medical artwork. She is a graduate of the University of Illinois at Chicago and certified as a medical illustrator by the Board of Certification of Medical Illustrators. Having a strong background in biological research, she specializes in 3D modeling, animation, and illustration to depict the aesthetics of science behind each idea.

Leah A. Lebowicz, M.S., CMI is a Clinical Assistant Professor in the Biomedical Visualization Graduate program (BVIS) at the University of Illinois at Chicago. As part of the BVIS faculty, Leah teaches the 3D modeling courses in the program, which include software such as Autodesk 3D Studio Max and Mudbox, Adobe Photoshop, Materialise Mimics and Pixologic ZBrush. She also co-teaches the Visual Learning and Visual Thinking I and II courses. She is interested in improving medical education with the use of 3D interactive programs, specifically in the field of embryology.

Leah has served as a research chair and committee member on numerous Master student research projects since 2012. The research topics she and her students have investigated have involved 3D modeling, 2D and 3D animation, interactive 
program design and development, virtual reality, embryology, medical education, paleontology reconstructions, and visualizing medical diagnostic procedures.

Christine D. Young, M.A, CMI, FAMI, is a Clinical Assistant Professor in the Biomedical Visualization Graduate Program at University of Illinois at Chicago. Christine mentors BVIS research projects as well as teaching Business Practices, Animation I, co-teaches Visual Learning \& Visual Thinking with Leah Lebowicz, and has developed curriculum for the Medical Legal Visualization course with Christa Wellman. In addition, she owns a small studio in Evanston, IL dedicated to biomedical science visualization.

Deborah M. Milkowski, Ph.D., is an Adjunct Clinical Assistant Professor in the Biomedical Visualization Graduate Program at the University of Illinois at Chicago and the Chief Scientific Officer at Jace Biomedical, Inc. 